$$
\text { EUI - Series C - } 6.2
$$

Flora (Ed.), Growth to Limits 
European University Institute Institut Universitaire Européen Europäisches Hochschulinstitut Istituto Universitario Europeo

\author{
Series C \\ Political and Social Sciences \\ Sciences Politiques et Sociales \\ Politik- und Sozialwissenschaften \\ Scienze Politiche e Sociali
}

6.2

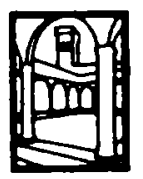

Badia Fiesolana - Firenze 


\title{
Growth to Limits
}

\section{The Western European Welfare States Since World War II}

\author{
Volume 2 \\ Germany, United Kingdom, Ireland, Italy
}

\author{
Edited by
}

Peter Flora

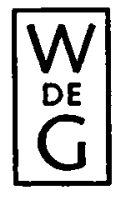

1986

Walter de Gruyter - Berlin - New York 
Library of Congress Cataloging-in-Publication Data

Growth to limits.

Contents: v. 1. Sweden, Norway, Finland, Denmark --

v. 2. Germany, United Kingdom, Ireland, Italy.

1. Europe--Social conditions--20th century. 2. Welfare state.

3. Europe--Politics and government--1945-

4. Europe--Economic conditions--1945- . I. Flora, Peter, 1944-

HN377.G76 $1986 \quad 306.7^{\prime} 094 \quad 86-19938$

ISBN 0-89925-266-4 (U.S. : v. 1)

\section{CIP-Kurztitelaufnabme der Deutschen Bibliothek}

Growth to limits : the Western European welfare states since World War II / ed. by Peter Flora. - Berlin; New York : de Gruyter (European University Institute : Ser. C, Political and social sciences ; 6)

NE: Flora, Peter [Hrsg.]; Istituto Universitario Europeo (Fiesole): European University Institute / C

Vol. 2. Germany, United Kingdom, Ireland, Italy. - 1986.

ISBN 3-11-011131-4

(C) Copyright 1986 by Walter de Gruyter \& Co., Berlin.

All rights reserved, including those of translation into foreign languages. No part of this book may be reproduced in any form - by photoprint, microfilm, or any other means - nor transmitted nor translated into a machine language without written permission from the publisher.

Dust Cover Design: Rudolf Hübler, Berlin. - Setting: Satz-Rechen-Zentrum, Berlin.

Printing: H. Heenemann GmbH \& Co., Berlin.

Binding: Verlagsbuchbinderei Dieter Mikolai, Berlin.

Printed in Germany. 